priority area for research and development to meet the clinical challenges posed by the scale of the infection in the UK. There has been a perceived lack of connection between clinicians and basic scientists working on HCV in the UK to address this problem. Aims: To create a multi-disciplinary consortium comprising clinicians and non-clinical scientists to encourage translational research. To establish a cohort of 10000 patients with HCV infection across the $\mathrm{UK}$, together with clinical database and biorepository- "HCV Research UK". To make this resource available to researchers, both academic/commercial, UK-based and abroad.

Methods Aims: Our objective has been to create a multi-disciplinary consortium comprising clinicians and non-clinical scientists to encourage translational research into the factors that determine outcome of infection, treatment response and disease progression. We aim to establish a cohort of 10000 patients with HCV infection from across the UK that is supported by the necessary systems to make clinical data and specimens available to academic and commercial researchers, both in the UK and abroad.

Results Progress: HCV Research UK has been funded by the Medical Research Foundation (£1.92 million) to establish an infrastructure that connects 18 clinical centres who will recruit $10000 \mathrm{HCV}$ infected patients. The key elements of the infrastructure are a bespoke clinical research database, which is linked to a biorepository of samples that will hold serum, PBMCs and DNA from patients. Access to data and samples is managed by a Tissue and Data Access Committee who have the authority to grant ethical approval for research using the resource. The study has been given CLRN portfolio status.

Conclusion Future plans: Recruitment of patients will begin in early 2012 and grant applications have been submitted to (1) Wellcome/ Department of Health Innovation Challenge fund (2) MRC call for Stratified Medicine (3) BLT Research call (4) NIHR Programme Development award schemes for research using the resource. It is hoped that portfolio support will provide a mechanism for new centres to join the consortium.

Competing interests None declared.

\section{PMO-183 ROLE OF IL28B POLYMORPHISM IN PREDICTION OF RESPONSE TO THERAPY IN PATIENTS WITH GENOTYPE 1 CHRONIC HEPATITIS C INFECTION}

doi:10.1136/gutjnl-2012-302514b.183

${ }^{1} Z$ Mustafa, ${ }^{* 2} \mathrm{D}$ Gaffney, ${ }^{1} \mathrm{E}$ Matthews, ${ }^{1} \mathrm{~S}$ Barclay, ${ }^{1} \mathrm{M}$ Priest, ${ }^{2} \mathrm{R}$ Spooner, ${ }^{1} \mathrm{P}$ R Mills. ${ }^{1}$ Gastroenterology, North Glasgow University Hospital, Glasgow, UK; ${ }^{2}$ Biochemistry, North Glasgow University Hospital, Glasgow, UK

Introduction Patients with chronic hepatitis $\mathrm{C}$ virus (HCV) infection have a variable response to antiviral therapy with pegylated interferon and ribavirin. Influences include age, gender, viral genotype, viral load, severity of liver disease and coinfection. Around $45 \%$ of patients with viral genotype 1 (G1) infection respond compared with $70 \%-80 \%$ with genotype $2 / 3$. Recently a human IL28B polymorphism has been found to predict response in patients with G1 infection. There is little data on this from Europe and a study of IL28B polymorphisms in patients with G1 infection treated in Glasgow was conducted.

Methods Sequential Caucasian patients with G1 chronic HCV who had been treated with combination antiviral therapy were studied. Responses were classified as sustained viral response (SVR), relapse (R) or non-responder (NR). None had coinfection. Data on age, gender, viral load, duration of therapy and severity of liver disease (Ishak fibrosis stage $<4$ or $\geq 4$ ) were collected. Individuals were genotyped for IL28B polymorphism rs12979860 using TaqMan ${ }^{\circledR}$, Drug Metabolism Genotyping Assays and reported as CC, CTor TT.
Results 63 patients were classified (number, mean age, females, advanced fibrosis) by treatment response as $\operatorname{SVR}(18,44,4,1), \mathrm{R}(20$, $46,8,8)$ and $\mathrm{NR}(25,46.4,4,10)$. Mean pre-treatment viral load was similar in the three groups $\left(5.2,5.4,5.8 \log _{10} \mathrm{IU} / \mathrm{ml}\right)$ and mean duration of therapy shorter for NR (46.2, 47.2, 8.4 weeks) who often fulfilled an early stopping rule. The IL28B genotype was highly predictive of response (Abstract PMO-183 table 1). CC individuals have a much greater likelihood $(p<0.002)$ of being in the SVR group than CT or TT individuals. Poorer response was also seen in patients with advanced fibrosis.

Abstract PM0-183 Table 1

\begin{tabular}{lllll}
\hline & Total & CC & CT & TT \\
\hline SVR & 18 & 12 & 4 & 2 \\
Relapse & 20 & 9 & 10 & 1 \\
Non-responder & 25 & 2 & 16 & 7 \\
\hline
\end{tabular}

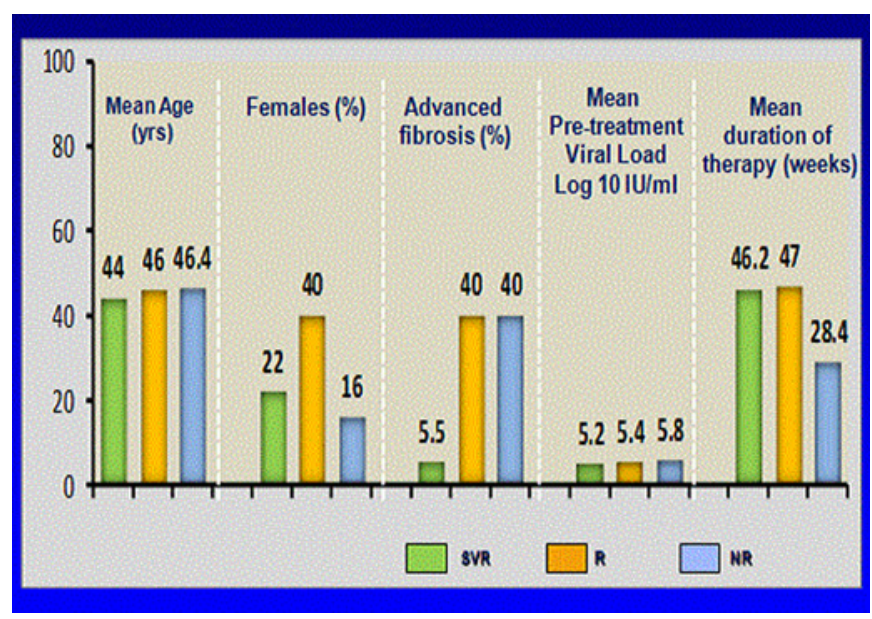

Abstract PM0-183 Figure 1 Characteristics of study subjects

Conclusion The IL28B polymorphism is a useful and cheap assay allowing some prediction of response to antiviral therapy in patients with G1 chronic HCV infection.

Competing interests None declared.

\section{Endoscopy I \\ PMO-184 COLONOSCOPIC TATTOOING OF COLORECTAL NEOPLASIA: A CHANGE IN PRACTICE}

doi:10.1136/gutjnl-2012-302514b.184

${ }^{1}$ A Brigic, ${ }^{*}{ }^{2} \mathrm{~J}$ Clarke, ${ }^{1} \mathrm{~A}$ Haycock, ${ }^{1} \mathrm{~S}$ Thomas-Gibson. ${ }^{1}$ Wolfson Endoscopy Unit, St. Mark's Hospital and Academic Institute, London, UK; ${ }^{2}$ Department of Surgery, St. Mark's Hospital and Academic Institute, London, UK

Introduction Quality Assurance Guidelines for colonoscopy in the Bowel Cancer Screening Programme recommend tattooing of all lesions that may require later surgical or endoscopic localisation, using local protocols as guidance. ${ }^{1}$ The St. Mark's Hospital colonoscopic tattooing protocol stated that all suspicious lesions should be tattooed, with the exception of those in the caecum and within $20 \mathrm{~cm}$ of the anal verge. Three tattoos should be placed $\left(120^{\circ}\right.$ apart, close to the lesion) and distal to lesions proximal to the splenic flexure (SpFlx). Left sided lesions should have tattoos placed proximal to the lesion. Our aim was to audit compliance with the tattooing protocol in patients undergoing surgery for colorectal neoplasia. 